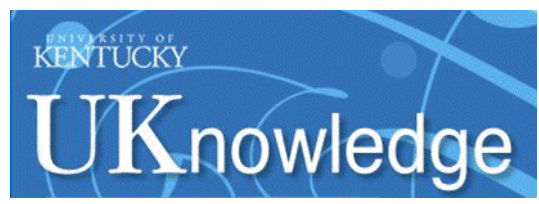

University of Kentucky

UKnowledge

Kentucky Tobacco Research and Development Center Faculty Publications

2-12-2009

\title{
Increased Hydrogen Production by Genetic Engineering of Escherichia coli
}

\author{
Zhanmin Fan \\ University of Kentucky
}

Ling Yuan

University of Kentucky, lyuan3@uky.edu

Ranjini Chatterjee

Farasis Energy, Inc.

Follow this and additional works at: https://uknowledge.uky.edu/ktrdc_facpub

Part of the Agriculture Commons, and the Plant Sciences Commons

Right click to open a feedback form in a new tab to let us know how this document benefits you.

\section{Repository Citation}

Fan, Zhanmin; Yuan, Ling; and Chatterjee, Ranjini, "Increased Hydrogen Production by Genetic Engineering of Escherichia coli" (2009). Kentucky Tobacco Research and Development Center Faculty Publications. 3. https://uknowledge.uky.edu/ktrdc_facpub/3

This Article is brought to you for free and open access by the Tobacco Research and Development at UKnowledge. It has been accepted for inclusion in Kentucky Tobacco Research and Development Center Faculty Publications by an authorized administrator of UKnowledge. For more information, please contact UKnowledge@lsv.uky.edu. 


\section{Increased Hydrogen Production by Genetic Engineering of Escherichia coli}

\section{Digital Object Identifier (DOI)}

http://dx.doi.org/10.1371/journal.pone.0004432

\section{Notes/Citation Information}

Published in PLoS One, v. 4, no. 2, p. 4432.

(C) 2009 Fan et al. This is an open-access article distributed under the terms of the Creative Commons Attribution License, which permits unrestricted use, distribution, and reproduction in any medium, provided the original author and source are credited. 


\title{
Increased Hydrogen Production by Genetic Engineering of Escherichia coli
}

\author{
Zhanmin Fan ${ }^{1}$, Ling Yuan ${ }^{1}$, Ranjini Chatterjee ${ }^{2 *}$
}

1 Department of Plant and Soil Sciences and Kentucky Tobacco Research and Development Center, University of Kentucky, Lexington, Kentucky, United States of America, 2 Farasis Energy, Inc., Hayward, California, United States of America

\begin{abstract}
Escherichia coli is capable of producing hydrogen under anaerobic growth conditions. Formate is converted to hydrogen in the fermenting cell by the formate hydrogenlyase enzyme system. The specific hydrogen yield from glucose was improved by the modification of transcriptional regulators and metabolic enzymes involved in the dissimilation of pyruvate and formate. The engineered $E$. coli strains ZF1 ( $\triangle$ focA; disrupted in a formate transporter gene) and ZF3 ( $\Delta$ narL; disrupted in a global transcriptional regulator gene) produced 14.9 , and $14.4 \mu$ mols of hydrogen $/ \mathrm{mg}$ of dry cell weight, respectively, compared to $9.8 \mu \mathrm{mols}$ of hydrogen/mg of dry cell weight generated by wild-type $E$. coli strain W3110. The molar yield of hydrogen for strain ZF3 was $0.96 \mathrm{mols}$ of hydrogen $/ \mathrm{mol}$ of glucose, compared to $0.54 \mathrm{mols}$ of hydrogen/mol of glucose for the wild-type $E$. coli strain. The expression of the global transcriptional regulator protein FNR at levels above natural abundance had a synergistic effect on increasing the hydrogen yield in the $\triangle$ focA genetic background. The modification of global transcriptional regulators to modulate the expression of multiple operons required for the biosynthesis of formate hydrogenlyase represents a practical approach to improve hydrogen production.
\end{abstract}

Citation: Fan Z, Yuan L, Chatterjee R (2009) Increased Hydrogen Production by Genetic Engineering of Escherichia coli. PLoS ONE 4(2): e4432. doi:10.1371/ journal.pone.0004432

Editor: Christophe Herman, Baylor College of Medicine, United States of America

Received October 9, 2008; Accepted November 24, 2008; Published February 12, 2009

Copyright: $(2009$ Fan et al. This is an open-access article distributed under the terms of the Creative Commons Attribution License, which permits unrestricted use, distribution, and reproduction in any medium, provided the original author and source are credited.

Funding: This work was supported by the Small Business Innovative Research grant DE-FG02-03ER83868 from the U.S. Department of Energy. The funders had no role in study design, data collection and analysis, decision to publish, or preparation of the manuscript.

Competing Interests: The authors have declared that no competing interests exist.

*E-mail: rjic@novozymes.com

\section{Introduction}

The present emphasis on the diversification of global energy sources, while reducing the consumption of fossil fuels, has placed renewed interest on hydrogen as an alternative fuel and its synthesis through microbe-mediated bioconversions. Microbes have evolved unique mechanisms for hydrogen generation, and some of these mechanisms are being explored for biotechnological applications including nitrogenase-mediated and fermentative hydrogen production [1]. Efforts to improve hydrogen production have been focused on pathway redirection, identification and engineering of oxygen-tolerant hydrogenases, improvement in hydrogen molar yields and development of efficient hydrogen separation techniques from bioreactor headspace [1-5].

The production of hydrogen by facultative anaerobic organisms such as $E$. coli is a characteristic of mixed acid fermentation. Under anaerobic conditions, the fermentation products comprise a mixture of ethanol, succinate, lactate, acetate, and formate (Fig. 1). Succinate is produced via the succinate pathway. A key reaction in this pathway is carboxylation of phosphoenolpyruvate (PEP) to the four-carbon compound oxaloacetate by phosphoenolpyruvate carboxylase (PEPG) [6]. PEP is also converted to pyruvate which is subsequently converted to acetyl-CoA and formate by pyruvate formate lyase (PFL), and to lactate by lactate dehydrogenase $(\mathrm{LDH})$. Formate hydrogenlyase (FHL) catalyzes the conversion of formate to carbon dioxide and hydrogen $[7,8]$. The intracellular level of formate is determined by the rates of biosynthesis and metabolism of formate, and also by formate transporter FocA which is a membrane protein involved in formate transport into and out of the cell [9]. Knockout of the foc $A$ gene results in intracellular accumulation of formate [10]. The FHL enzyme complex is comprised of formate dehydrogenase $\mathrm{H}$ $\left(\mathrm{FDH}_{\mathrm{H}}\right)$, and a hydrogen-evolving hydrogenase (hydrogenase 3) $[11,12]$. The biosynthesis of PFL and FHL are up-regulated by the action of multiple transcriptional regulators including the global transcriptional factors Fnr, ArcAB and integration host factor (IHF) [13-15], and repressed by the dual transcriptional regulator NarL (Table 1)[16]. The transcription of the fhl regulon is regulated by the primary and secondary transcriptional activator FHLA and ModE $[17,18]$. The expression of fhlA is activated by Fnr in response to the cellular redox state $[19,20]$. The biosynthesis of $\mathrm{FDH}_{\mathrm{H}}$ also requires the expression of $\mathrm{sel} C$ gene which encodes a tRNA for the incorporation of selenocysteine to $\mathrm{FDH}_{\mathrm{H}}($ Table 1) $[8,11,21]$.

Successful efforts to redirect pyruvate towards hydrogen in $E$. coli, have involved the up-regulation of the FHL complex and the disruption of pathways competing for pyruvate [4,22-24]. It is estimated that over 50 genes distributed across at least 20 distinct genetic loci define fermentative hydrogen metabolism in E. coli [8]. In this study, our strategy involves the systematic modification of multiple, discrete, metablic segments that include global regulatory, transport, and auxiliary components required for FHL biosynthesis, processing and assembly. The modification of key regulatory elements represents a practical strategy for the coordinated engineering of genes and operons that perform distinct biochemical functions related to the production of 


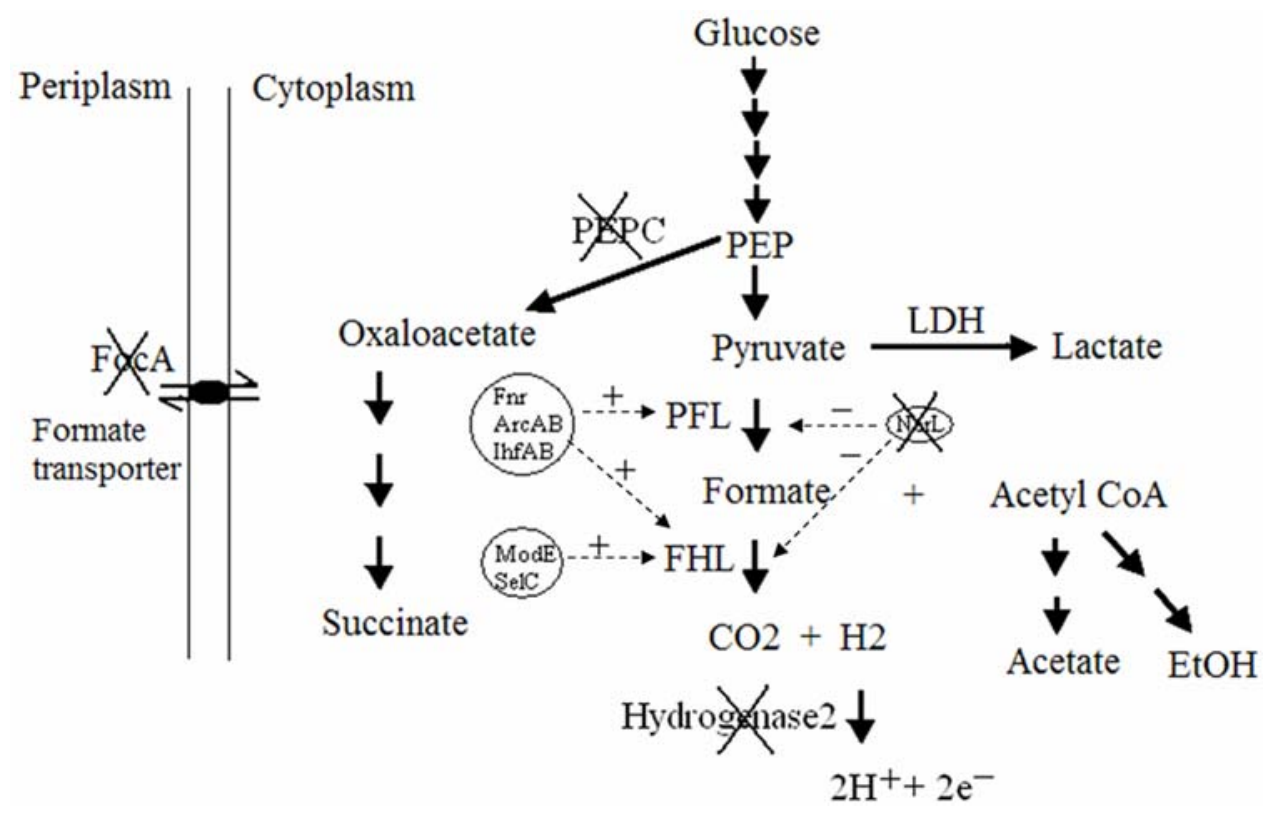

Figure 1. The genetic modification of metabolic pathways and regulatory components for hydrogen production in $E$. coli. The metabolic flows are indicated by solid arrows. Some key enzyme systems are labeled. The key global regulators and their regulatory targets are circled and indicated by dashed arrows, respectively. Pluses (+) represent activation, and minuses $(-)$ represent repression. Crosses $(\mathrm{X})$ indicate chromosomal gene disruptions.

doi:10.1371/journal.pone.0004432.g001

hydrogen. This approach has the potential to achieve the balance of cofactor and precursor supply pathways with the biosynthesis of structural polypeptides without placing an unnecessary metabolic burden on the cells [25]. Here, we describe increases in specific and molar yields of hydrogen achieved by the modification of the foc $A$, $p p c$, narL, and fnr genes involved in precursor transport and metabolism, and the global regulation of fermentative hydrogen production by E. coli.

\section{Results and Discussion}

\section{Strategy for genetic modification}

The fermentation pathways initiate by the non-oxidative cleavage of pyruvate to acetyl-CoA and formate by PFL; formate is subsequently metabolized to hydrogen and carbon dioxide by FHL. Our strategy for improving fermentative hydrogen production involved the modification of precursor transport and metabolism, and of regulatory elements, to direct the flow of the two key precursor metabolites pyruvate and formate towards hydrogen, and achieve increased expression of both structural and auxiliary components of the FHL enzyme system. The biochemical reactions and regulatory elements that were modified by the inactivation of specific chromosomal genes, or by plasmid-directed gene overexpression are illustrated schematically in Figure 1; the functions of the corresponding gene products are summarized in Table 1.

The inactivation of the four selected genes in the chromosome of $E$. coli strain W3110 was hypothesized to increase the cellular concentrations of pyruvate and formate, reduce the re-oxidation of evolved hydrogen, and relieve the repression of genes required for PFL and FHL biosynthesis. The $p p c$ gene encoding phosphoenolpyruvate carboxylase (PEPG) was disrupted to increase cellular pyruvate concentrations available for formate biosynthesis $[6,7]$. The gene encoding the formate transporter, foc $A$, was inactivated to elevate intra-cellular formate levels for FHL biosynthesis and

Table 1. Genes engineered in this study, and their functions.

\begin{tabular}{|c|c|}
\hline Gene & Function of gene product \\
\hline focA & Transmembrane protein involved in formate transport. \\
\hline hybC & Large catalytic subunit of hydrogenase 2 ; catalyzes dihydrogen oxidation. \\
\hline narl & $\begin{array}{l}\text { Global transcriptional regulator of anaerobic metabolism; represses the transcription of nik operon genes, fdhF and } p f l B \text { genes; activates the } \\
\text { transcription of formate dehydrogenase } \mathrm{N} \text {, which couples formate oxidation to nitrate reduction. }\end{array}$ \\
\hline ppc & Phosphoenolpyruvate carboxylase (PEPC) catalyzes the carboxylation of phosphoenolpyruvate (PEP) to oxaloacetate (OAA). \\
\hline fnr & Global transcriptional regulator of anaerobic metabolism; activator of $f h l A$ and $p f l B$ genes, and of the hyp, nik and moa operon genes. \\
\hline $\operatorname{arc} A B$ & Global transcriptional regulator of aerobic and anaerobic metabolism; activator of pflB, and repressor of the hyb and hya operon genes. \\
\hline ihfAB & Global transcriptional regulator activating the $p f I B$ and $f h I A$ genes, and genes of the hyc and hyp operons. \\
\hline $\bmod E$ & Secondary transcriptional activator of the $f h /$ regulon. \\
\hline selC & tRNA for selenocysteine incorporation into FDH-F polypeptide of FHL. \\
\hline
\end{tabular}




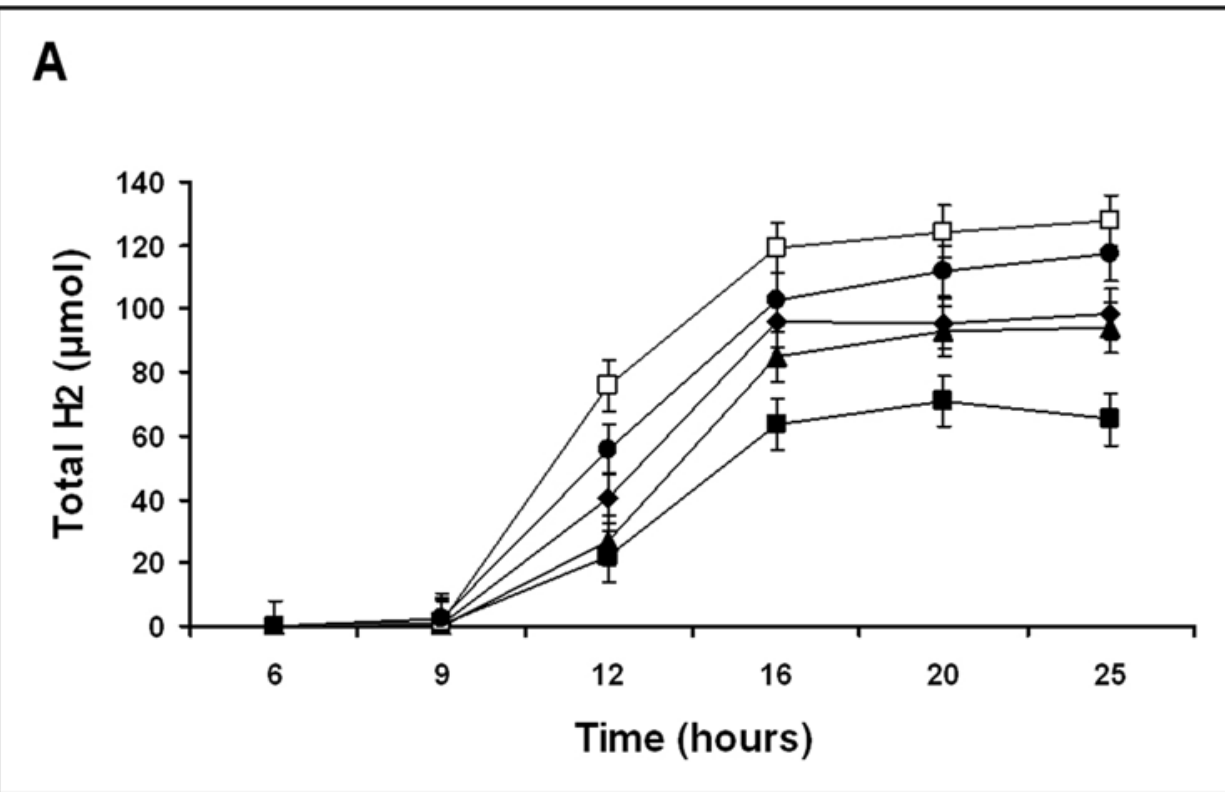

B

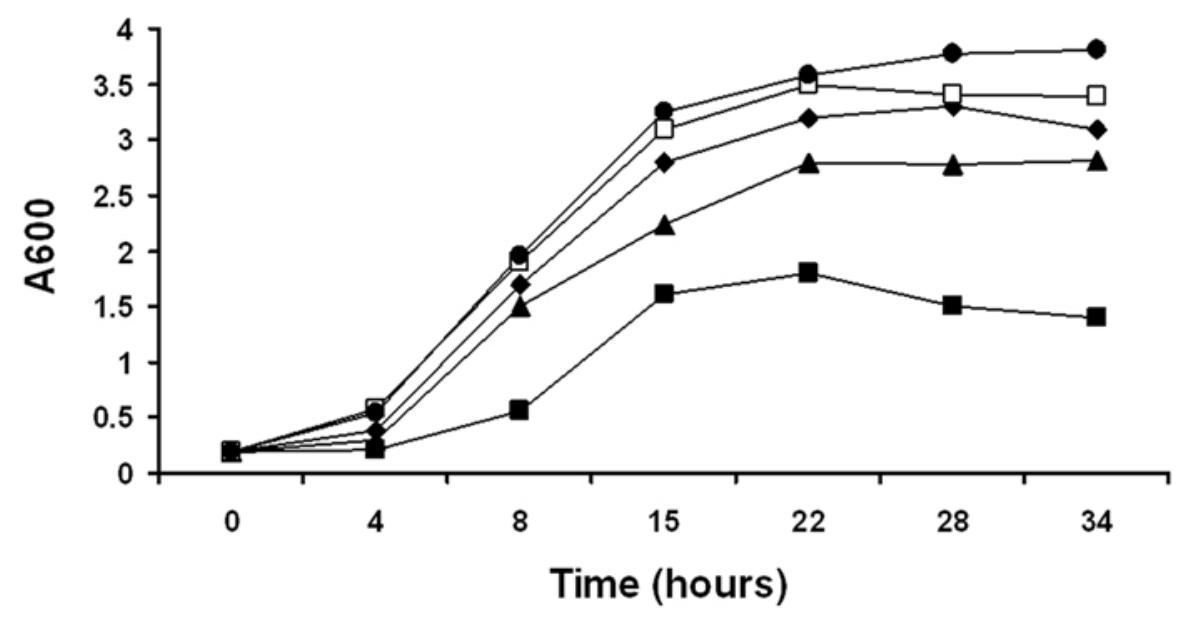

Figure 2. Hydrogen production and anaerobic growth rates of WT and single gene inactivated $E$. coli strains. Hydrogen production rate $\left(\mathrm{A}, \mu \mathrm{mol}\right.$ of $\left.\mathrm{H}_{2}\right)$ and anaerobic growth rate $(\mathrm{B}, \mathrm{A600})$ by strains W3110 $(\bullet), \mathrm{ZF} 1(\mathbf{\square}), \mathrm{ZF} 2(\boldsymbol{\Lambda}), \mathrm{ZF3}(\square), \mathrm{ZF4}(\bullet)$ on M9 medium supplemented with $1 \%$ $(\mathrm{w} / \mathrm{v})$ glucose.

doi:10.1371/journal.pone.0004432.g002

conversion to hydrogen [10]. E. coli synthesizes two uptake hydrogenases, hydrogenasel and 2, which oxidize hydrogen to protons with the release of electrons. In this study the hybC gene, which encodes the large catalytic subunit of hydrogenase 2, was inactivated to reduce the oxidation of hydrogen [26]. The global transcriptional regulator NarL represses transcription of the structural genes of FHL $(f d h F)$, PFL $(p f l B)$, and of the genes of the nik operon, which are required for the transport and metabolism of nickel for Hydrogenase cofactor biosynthesis [16]. To eliminate the repression described, the nar $L$ gene was inactivated.

The global transcriptional regulators Fnr, ArcAB and IhfAB activate multiple genes of anaerobic pyruvate and formate metabolism. Targets for these global regulators include fhlA the specific transcriptional activator of the $f h l$ regulon), $p f l B$, and the genes of the moa, nik and hyp operons required for the biosynthesis of the molybdopterin and Ni-Fe cofactors of FHL (Table 1) $[15,19,27,28]$. The effects on hydrogen yield of multi-copy expression of the global regulators $f n r, \operatorname{arc} A B$ and $\ln f A B$, as well as $M o d E$ (the secondary transcriptional activator of the $f h l$ regulon), and the tRNA for selenocysteine incorporation (selC), were examined in wild-type $\mathrm{W} 3110$ and $\Delta f o c A(\mathrm{ZF} 1)$ strains.

\section{Increased hydrogen yields by gene-inactivated $E$. coli} strains

The rate of hydrogen production and of growth by wild-type strain W3110, and four strains containing specific chromosomal gene disruptions were examined (Figures 2A and 2B). The 
Table 2. Hydrogen yields for strain W3110 with or without defined chromosomal gene disruptions.

\begin{tabular}{|c|c|c|}
\hline Strains & Hydrogen yield ( $\mu \mathrm{mol} / \mathrm{mg}$ dry cell mass) & Hydrogen yield from glucose ( $\mathrm{mol} / \mathrm{mol}$ glucose $)$ \\
\hline W3110 & $9.80 \pm 0.72$ & $0.54 \pm 0.04$ \\
\hline ZF1 (W3310 $f \circ c A)$ & $14.85 \pm 0.83$ & $0.63 \pm 0.04$ \\
\hline ZF2 (W3310AhybC) & $12.14 \pm 0.88$ & $0.70 \pm 0.05$ \\
\hline 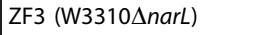 & $14.39 \pm 0.90$ & $0.96 \pm 0.07$ \\
\hline ZF4 (W3310 $p p p C)$ & $11.20 \pm 0.89$ & $0.73 \pm 0.05$ \\
\hline ZF5 (W3310 ffocA $h y b C$ ) & $8.65 \pm 0.78$ & Not determined \\
\hline
\end{tabular}

The hydrogen yields were determined following 17 hours of anaerobic growth on M9 medium containing $415 \mu$ mols of glucose.

doi:10.1371/journal.pone.0004432.t002

maximum rate of hydrogen generation occurred between 9 and 16 hours following inoculation of M9 medium under the

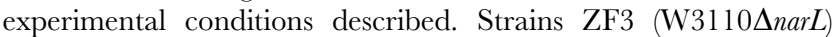
and ZF4 (W3110 $\Delta p p c)$ exhibited the highest rates of hydrogen production, and had accumulated about $20 \%$ to $30 \%$ more hydrogen in the culture headspace than W3110 in 25 hours (Figure 2A). Strain ZF1 (W3110 $\Delta$ focA) exhibited a slightly slower rate of hydrogen generation than W3110 and accumulated 33\% less hydrogen by the end of the fermentation experiment. Strains ZF2 (W3110 $h y b C$ ) and W3110 had comparable rates of hydrogen formation to each other. The slower rate of hydrogen generation observed in the later stages of fermentation correlated with an increased accumulation of biomass, possibly resulting from the hydrogen oxidizing activity of uptake hydrogenases 1, 2, and/or 4, generating protons and electrons for cellular ATP synthesis, or by inhibition of hydrogenase 3 by the evolved hydrogen that accumulated in culture headspace $[8,26]$. The complete consumption of glucose accompanied by reduced glycolytic flux to pyruvate and subsequently to formate could account for the simultaneous reductions in growth and $\mathrm{H}_{2}$ production rates. Strains W3110,

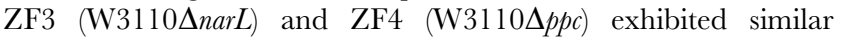
generation times and reached similar final cell densities. Strain ZF2 (W3110 $h y b C$ ) grew at a slightly slower rate than W3110, while strain ZF1 (W3110 $f$ focA) grew at a slower rate than W3110 and reached a $50 \%$ lower final culture density (Figure $2 \mathrm{~B}$ ). The lower growth rates of strains ZF1 and ZF2 might result from the increased acidification of cellular cytoplasm from the disruption of formate export, and by the decrease in cellular ATP synthesis by the disruption of an uptake hydrogenase, respectively. The rate of anaerobic growth and of hydrogen production by the strains in TYP medium followed a profile similar to that seen in M9-glucose medium, with the highest rates of hydrogen production occurring between 4 and 7 hours of fermentation. The culture generation times were increased by about 2.5-fold in TYP medium, with $\mathrm{A}_{600}$ values reaching about 6.5 by 10 hours (data not shown).

The specific hydrogen yields were determined for W3110 and the gene-inactivated strains at 17 hours following inoculation of M9-glucose medium (Table 2). All four gene-inactivated strains exhibited $14 \%$ to $50 \%$ higher specific hydrogen yields compared

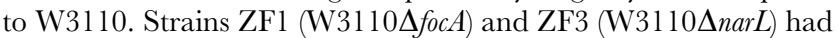
specific hydrogen yields of approximately $14 \mu \mathrm{mols} / \mathrm{mg}$ dry cell mass, representing an increase of about $46 \%$ over the yield by W3110. The increased hydrogen yield by strain ZF1 (W3110 $\Delta f o c A)$ is consistent with the hypothesis that an increase in the level of intracellular formate can either lead to enhanced synthesis of the FHL polypeptides via the transcriptional activator FhlA, or elevate the available substrate pool for FHL [29,30]. The negative effect on growth rates by the focA deletion might be alleviated if the intracellular formate can be efficiently metabolized by FHL (further described below). The $50 \%$ increase in specific hydrogen yield by strain ZF3 (W31104narL) showed that the elimination of transcriptional repression of nik operon, and of $f d h F$ and $p f l B$ genes by the NarL, results in the increased pyruvate metabolism towards hydrogen $[8,31]$.

The specific hydrogen yield of strain ZF2 (W3110 4 hybC) was $22 \%$ higher than W3110 (Table 2). Of the three E. coli uptake hydrogenases, hydrogenase 2 is believed to be the more active one based on in vitro kinetic studies [8]. The double mutant ZF5 (W3110 $\Delta$ foc $A \Delta h y b C$ ) did not produce more hydrogen than the

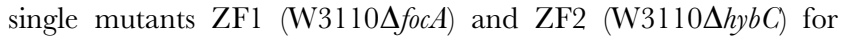
reasons that remain unclear. Inactivation of the metabolic enzyme PEPC resulted in approximately $14 \%$ increase in specific hydrogen yields.

If all of the pyruvate is directed towards the PFL reaction the theoretical maximum yield of hydrogen from a hexose sugar is

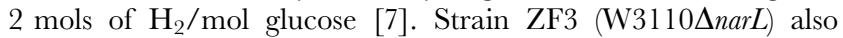
displayed the best molar yield of hydrogen (mols of $\mathrm{H}_{2} / \mathrm{mol}$ of glucose) of approximately 0.96 compared to 0.54 for W3110 (Table 2). The utility of eliminating transcriptional repression of multiple operons in anaerobic metabolism is highlighted by the effects of the narL gene deletion. This deletion confers a 1.5 -fold increase in specific hydrogen yield and a 2-fold increase in molar hydrogen yield, while retaining the growth characteristics of the wild-type strain W3110.

To examine the partitioning of glucose in the two high hydrogen yielding strains the distribution of fermentation products

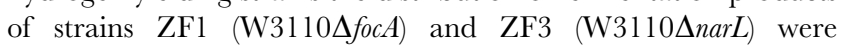
compared to that of W3110 (Table 3). These two strains carried out a balanced fermentation, with over $90 \%$ of the added glucose converted to products. No remaining glucose was detected in the medium by HPLC and spectrophotometric assays. Medium from

Table 3. The fermentation products of strains W3110, ZF1

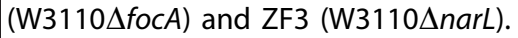

\begin{tabular}{|c|c|c|c|}
\hline & W3110 & ZF1 (W3310 $\Delta f o c A)$ & ZF3 (W3310 \\
\hline Hydrogen ( $\mu \mathrm{mol})$ & $91.0 \pm 1.34$ & $122.84 \pm 1.89$ & $118.0 \pm 1.42$ \\
\hline Formate $(\mu \mathrm{mol})$ & $114.24 \pm 1.32$ & $66.86 \pm 0.87$ & $112.83 \pm 1.26$ \\
\hline Lactate $(\mu \mathrm{mol})$ & $158.92 \pm 1.47$ & $218.40 \pm 1.88$ & $159.66 \pm 1.21$ \\
\hline Acetate $(\mu \mathrm{mol})$ & $106.36 \pm 1.17$ & $107.96 \pm 1.15$ & $109.14 \pm 1.15$ \\
\hline Succinate $(\mu \mathrm{mol})$ & $41.48 \pm 0.76$ & $42.85 \pm 0.72$ & $43.46 \pm 0.81$ \\
\hline Ethanol $(\mu \mathrm{mol})$ & $344.0 \pm 2.87$ & $285.43 \pm 2.25$ & $229.0 \pm 1.87$ \\
\hline
\end{tabular}

The concentrations of metabolites in culture media were determined following 34 hours of anaerobic growth on M9 medium containing $830 \mu$ mols of glucose. doi:10.1371/journal.pone.0004432.t003 
Table 4. Hydrogen yields for strain W3110 and ZF1 (W3110 $\Delta$ focA) when expressing plasmid-borne genes.

\begin{tabular}{|c|c|c|}
\hline Strain & Genotype & $\begin{array}{l}\text { Hydrogen yield } \\
\text { ( } \mu \mathrm{mol} / \mathrm{mg} \text { dry cell mass) }\end{array}$ \\
\hline ZF6 & W3110/pACYC177L & $1.07 \pm 0.03$ \\
\hline ZF7 & W3110/pfnr & $6.23 \pm 0.45$ \\
\hline ZF8 & W3110/parcAB & $1.11 \pm 0.04$ \\
\hline ZF9 & W3110/pihfAB & $1.14 \pm 0.04$ \\
\hline ZF10 & W3110/pmodE & $1.27 \pm 0.06$ \\
\hline ZF11 & W3110/pselC & $1.81 \pm 0.06$ \\
\hline ZF12 & W3110 $f \circ c A / p A C Y C 177 L$ & $0.00 \pm 0.00$ \\
\hline ZF13 & W3110 $\Delta$ focA/pfnr & $10.32 \pm 0.58$ \\
\hline ZF14 & W3110 $\triangle f o c A / p a r c A B$ & $0.19 \pm 0.02$ \\
\hline ZF15 & W3110 $\triangle f o c A /$ pihfAB & $0.61 \pm 0.03$ \\
\hline ZF16 & W3110 $\Delta f o c A / p m o d E$ & $0.63 \pm 0.03$ \\
\hline ZF17 & W3110 $A$ focA/pselC & $1.19 \pm 0.06$ \\
\hline
\end{tabular}

The specific hydrogen yields were determined following 4 hours of induction on TYP medium. TYP medium was supplemented with glucose at a final concentration of $0.5 \%(\mathrm{w} / \mathrm{v})$ for this experiment

doi:10.1371/journal.pone.0004432.t004

strain ZF1 (W3110 $\mathrm{AfocA}$ ) contained 40\% less formate compared to strains W3110 and ZF3 (W3110 AnarL), consistent with less formate being secreted from the cell due to the disruption of the channel for formate. Lactate formation was increased by about $37 \%$ in strain ZF1 (W3110 2 focA) compared to W3110, perhaps from the efficient induction of $l d h A$ due to lower intra-cellular $\mathrm{pH}$ from formate accumulation, or by the inhibition of PFL activity and/or biosynthesis by formate. The formation of ethanol was lowered by $17 \%$ and $33 \%$ in strains ZF1 (W3110 $\Delta$ focA) and ZF3 (W3110 narL), respectively, compared to that of W3110. These two strains accumulated succinate and acetate to levels similar to those of strain W3110.

\section{The effect of multi-copy gene expression on hydrogen yields}

Recent reports have described improvements in fermentative hydrogen yields in $E$. coli through the modification of the specific transcriptional activator, and repressor proteins of the FHL system, FhlA and HycA, respectively [22]. The three global regulators Fnr, ArcAB and IhfAB modulate the expression of multiple genes involved in the anaerobic metabolism of pyruvate in $E$. coli $[9,15,19,27,28]$. The impact on fermentative hydrogen yields by the over-production of the Fnr, ArcAB, and IhfAB proteins, and of ModE which is the secondary transcriptional activator of the hyc operon, was examined in strains W3110 and ZF1 (W3110 $\Delta f o c A)$. Strain ZF1 (W3110 $\Delta f o c A$ ) was selected because an increased level of cellular formate, combined with the overexpression of formate-metabolizing genes might have a synergistic effect on hydrogen formation. The specific hydrogen yields following induction of the plasmid-bearing strains are shown in Table 4. Strain ZF7, over-expressing of the fnr gene in W3110 background, exhibited an approximately 5.5-fold increase in specific hydrogen yield compared to the control strain ZF6 (containing the plasmid without promoter-driven gene expression). Over-expression of the $f n r$ gene in the $\Delta f o c A$ genetic background (strain ZF13) resulted in the generation of $10 \mu \mathrm{mol}$ of $\mathrm{H}_{2} / \mathrm{mg}$ of dry cell mass compared to $6 \mu \mathrm{mol}$ of $\mathrm{H}_{2} / \mathrm{mg}$ of dry cell mass obtained with over-expression of the $f n r$ gene in the wild-type
W3110 background (strain ZF7) in TYP medium. When induced in M9-glucose medium for 8 hours, strains ZF7 and ZF13 accumulated approximately $24 \mu \mathrm{mol}$ of $\mathrm{H}_{2} / \mathrm{mg}$ of dry cell mass and $34 \mu \mathrm{mol}$ of $\mathrm{H}_{2} / \mathrm{mg}$ of dry cell mass, respectively (data not shown). Over-expression of the global regulators ArcAB and IhfAB, and of the secondary transcriptional activator ModE, had no discernible effects on hydrogen yields in either W3110 or ZF1 strains in TYP or in M9-glucose medium. The synergistic effect on increased hydrogen yield by the over-expression of Fnr in the $\triangle f o c A$ genetic background suggests that genetic modifications to simultaneously increase both substrate and enzyme levels for hydrogen production are desirable to prevent the accumulation of formate inside the cell. These results show that specific genetic backgrounds provide additive effects on hydrogen yield when combined with increased gene dosage of particular metabolic enzymes or regulatory components.

While the Fnr protein activates transcription of operons involved in nickel and molybdenum metabolism for FHL biosynthesis [32], it has not been shown to affect the transcription of genes involved in selenium metabolism. To determine whether selenium metabolism is limiting to hydrogen production, the selC gene, encoding the tRNA for selenocysteine incorporation into the $\mathrm{FDH}_{\mathrm{H}}$ polypeptide, was over-expressed in strains W3110 and ZF1 (W3110 $\Delta$ focA). An approximately $63 \%$ increase in hydrogen yield was observed in strain ZF11 which expresses multiple copies of the selC gene compared to the negative control, strain ZF6 (Table 4). These results illustrate that hydrogen yields can be increased by improving the efficiency of a pathway for cofactor production for the FHL enzyme system.

One approach to obtaining sustained increases in fermentative hydrogen-yields will be to direct and improve the metabolism of pyruvate and formate towards hydrogen under anaerobic conditions. Genetic modifications that have led to increased hydrogen yields in $E$. coli have thus far focused on the disruption of key metabolic genes of the mixed acid fermentation pathways of E. coli, and of specific regulators of the FHL system. This report represents the first example of increased hydrogen production achieved through the modification of two global transcriptional regulators, a cofactor biosynthetic gene, and a transporter for formate. The construction of $E$. coli strains with specific chromosomal gene deletions, have revealed three unique genetic backgrounds that improve fermentative hydrogen production: inactivation of the foc $A$, $n a r L$, and $p p c$ genes lead to increased hydrogen yields over that of wild-type $E$. coli. The metabolism of selenium is one of several metabolic pathways required for the processing of metal ions for FHL biosynthesis. The improvement in hydrogen yield observed by the increased expression of the tRNA for selenocysteine suggests that cofactor biosynthesis pathways could be useful targets for modification. The increased hydrogen yields obtained by overexpression of the fnr gene, indicates that the anaerobic metabolism of pyruvate and formate can be coordinately up-regulated for improved flux towards hydrogen.

The results presented in this work illustrate the utility of modifying transport, regulatory, and auxiliary pathways related to FHL biosynthesis and function, to achieve increased hydrogen yields. The future application of genome engineering formats in combination with global gene expression analysis to E. coli will enable the identification of novel genes and metabolic functions underlying the improved hydrogen producing phenotype [25,33]. Similar approaches might be extended to enteric microbes that contain the FHL enzyme system to improve hydrogen production from alternate carbon sources, and for the simultaneous production of value-added chemical entities derived from the anaerobic metabolism of pyruvate. 


\section{Materials and Methods}

\section{Genetic manipulations}

All strains and plasmids used in this study are listed in Table 5, and PCR primers used are shown in Table 6. The disruption of chromosomal genes in E. coli K-12 strain W3110 was performed by the method of Datsenko and Wanner [34]. Plasmid pKD13 was used as the template for amplification of the FRT-flanked $\mathrm{Km}^{\mathrm{R}}$ gene with primers containing 44 to 50 nucleotide homology extensions. Plasmids pKD46 and pCP20 were used for the replacement of the target gene with the $\mathrm{Km}^{\mathrm{R}}$ gene, and for subsequent $\mathrm{Km}^{\mathrm{R}}$ gene removal, respectively. Primer pairs, focA$\mathrm{H} 2 \mathrm{P} 4 /$ focA-H1P1, hy $b C-\mathrm{H} 2 \mathrm{P} 4 /$ hy $b C-\mathrm{H} 1 \mathrm{P} 1$, nar $-\mathrm{H} 2 \mathrm{P} 4 /$ nar $-\mathrm{H} 1 \mathrm{P} 1$, and $p p c-\mathrm{H} 2 \mathrm{P} 4 / p p c-\mathrm{H} 1 \mathrm{P} 1$ were used for the disruption of the focA, $h y b C$, narL, and $p p c$ genes, respectively. The entire coding sequences of the $h y b C$, narL, and $p p c$ genes were deleted, while only 589 nucleotides at the $5^{\prime}$ terminal sequence of the foc $A$ gene were deleted since the sequence downstream of it overlaps the $p f l$ operon. The gene disruptions were verified by PCR analyses of genomic DNA using the primers $\mathrm{k} 1$ and $\mathrm{k} 2$ to the $\mathrm{Km}^{\mathrm{R}}$ gene, and the FW and RV primers to the flanking sequences of the disrupted genes.

The plasmid pACYC177 (Invitrogen) was used for the multicopy expression of the $E$. coli genes fnr, $\operatorname{arc} A B$, ihf $A B$, modE, and selC. The genes were amplified by PCR, using Pf $550^{\text {TM }}$ DNA polymerase (Invitrogen) to produce blunt ended PCR products, from genomic DNA of $E$. coli strain W3110. For the construction of pfnr, pmodE and pselC, the lac $Z$ promoter sequence from the pGEM $^{\circledR}$-T easy vector (Promega) was transferred into pAYCY 177 by amplification using primers Plac-BamHI-FW and Plac-PstI-RV, digestion with BamHI and Pst I, and ligation into the corresponding sites of pACYC177. The modified pACYC177 vector was designated pACYC177L. The $f n r, \operatorname{modE}$, and $s e l C$ genes were amplified with primer pairs: $f n r-\mathrm{FW} / f n r-\mathrm{RV}, \operatorname{modE}-\mathrm{FW} / \bmod E-\mathrm{RV}$ and $s e l C$-FW/selC-RV, respectively, digested with $P s t \mathrm{I}$ and $F s p \mathrm{I}$, and ligated into the corresponding sites of pACYC177L. For the construction of parcAB and pihfAB, primer Plac-ScaI-RV was used instead of primer Plac-PstI-RV for the amplification of the lacZ promotor, and ScaI was used for digestion of the PCR fragment. This modified pACYC177 vector was designated as pACYC177L1. The $\operatorname{arc} B$ and $i h f B$ genes were amplified using primer pairs $\operatorname{arcB}-X b a \mathrm{I}-\mathrm{FW} / \operatorname{arcB}-B g I \mathrm{I}-\mathrm{RV}$ and $i h f B-X b a \mathrm{I}-\mathrm{FW} / i h f B$ $B g I \mathrm{I}-\mathrm{RV}$, respectively. The two forward primers contained a $40-$ nucleotide linker sequence from genomic DNA of W3110. The PCR products were digested with $B g I \mathrm{I}$, and ligated to the $F s p \mathrm{I} / B g I \mathrm{I}$ site of pACYC177L1. Finally, the $\operatorname{arc} A$ and ihfA genes were amplified using primer pairs $\operatorname{arc} A-\mathrm{FW} / \operatorname{arc} A-X b a \mathrm{I}-\mathrm{RV}$ and ihfAFW/ihfA-XbaI-RV, digested with $X b a \mathrm{I}$, and ligated to $S c a \mathrm{I} / X b a \mathrm{I}$ site of pACYG177L1.

\section{Bacterial strains, media, and growth conditions}

All strains of E. coli used in this study are listed in Table 5. E. coli strains were routinely cultured at $37^{\circ} \mathrm{C}$ in either phosphatebuffered rich medium TYP $(10 \mathrm{~g} / \mathrm{L}$ tryptone, $5 \mathrm{~g} / \mathrm{L}$ yeast extract,

Table 5. E. coli strains and plasmids used in this study.

\begin{tabular}{|c|c|c|}
\hline Strains and plasmids & Genotype or description & Reference or source \\
\hline \multicolumn{3}{|l|}{ Strains } \\
\hline W3110 & F-I-IN $(r r n D-r r n E) 1 r p h-1$ & CGSC\# 4470 \\
\hline ZF1 & W3110 $\triangle$ focA & This work \\
\hline ZF2 & W3110 & This work \\
\hline ZF3 & 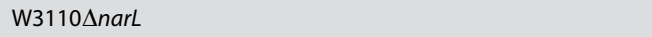 & This work \\
\hline ZF4 & W3110 $p p p c$ & This work \\
\hline ZF5 & W3110 $\Delta$ focA $\Delta h y b C$ & This work \\
\hline ZF6 & W3110 containing pACYC177L & This work \\
\hline ZF7 & W3110 containing pfnr & This work \\
\hline ZF8 & W3110 containing parcAB & This work \\
\hline ZF9 & W3110 containing pihfAB & This work \\
\hline ZF10 & W3110 containing pmodE & This work \\
\hline ZF11 & W3110 containing selC & This work \\
\hline ZF12 & ZF1containing pACYC177L & This work \\
\hline ZF13 & ZF1 containing pfnr & This work \\
\hline ZF14 & ZF1 containing parcAB & This work \\
\hline ZF15 & ZF1 containing pihf $A B$ & This work \\
\hline ZF16 & ZF1 containing pmodE & This work \\
\hline ZF17 & ZF1 containing pselC & This work \\
\hline \multicolumn{3}{|l|}{ Plasmids } \\
\hline pfnr & pACYC with LacZ promoter and W3110 fnr gene & This work \\
\hline parcAB & pACYC with LacZ promoter and W3110 arcA and $\operatorname{arc} B$ genes & This work \\
\hline pihfAB & pACYC with LacZ promoter and W3110 ihfA and ihfB genes & This work \\
\hline pmodE & pACYC with LacZ promoter and W3110 ihfA and ihfB genes & This work \\
\hline pselC & pACYC with LaCZ promoter and W3110 selC gene & This work \\
\hline
\end{tabular}


Table 6. Primers used in this study.

\begin{tabular}{|c|c|c|}
\hline Primer & Sequence & Source \\
\hline fOCA-H2P4 & 5'-CTTTGTTAGTATCTCGTCGCCGACTTAATAAAGAGAGAGTTAGTATTCCGGGGATCCGTCGACC-3' & This work \\
\hline fOCA-H1P1 & 5'-GATACTGTGCTCAAAACCGCTGGCAACAAACATCGCGACCGGCAGTGTAGGCTGGAGCTGCTTC-3' & This work \\
\hline focA-FW & 5'-GCCAGGCGAGATATGATCTA-3' & This work \\
\hline fOCA-RV & 5'-CTGCGTACTTCGACAACCAT-3' & This work \\
\hline hybC- $\mathrm{H} 2 \mathrm{P} 4$ & 5'-CCTTTAAAACAAAACGATCATAATCGTCATGAGGCGAGCAAAGCATGAGCATTCCGGGGATCCGTCGACC-3' & This work \\
\hline hybC-H1P1 & 5'-ATCGGTCAGCAAAATATTGCCGACCCCTAAGACTAAAATACGCATTACAGGTGTAGGCTGGAGCTGCTTC-3' & This work \\
\hline hybC-FW & 5'-GTCAGCACTGAGCGCACTGT-3' & This work \\
\hline hybC-RV & 5'-CAACGCCGGAACTTCTTCAT-3' & This work \\
\hline nar- $-\mathrm{H} 2 \mathrm{P} 4$ & 5'-CACATTCATTAAGGTTATTGCTCATTTAAAGCCTGAAGGAAGAGGTTTACATTCCGGGGATCCGTCGACC-3' & This work \\
\hline nar-H1P1 & 5'-TTCGCAGCATCGGGTGATCGTCAATCAGCAGGATAGTAGCCGGTTCCTGAGTGTAGGCTGGAGCTGCTTC-3' & This work \\
\hline nar-FW & 5'-GCCAATAGCCGAGAATACTG-3' & This work \\
\hline nar-RV & 5'-GACTCCGCCAGTTCAATACC-3' & This work \\
\hline ppc- $\mathrm{H} 2 \mathrm{P} 4$ & 5'-CGTGAAGGATACAGGGCTATCAAACGATAAGATGGGGTGTCTGGGGTAATATTCCGGGGATCCGTCGACC-3' & This work \\
\hline ppc-H1P1 & 5'-ATTTCAGAAAACCCTCGCGCAAAAGCACGAGGGTTTGCAGAAGAGGAAGAGTGTAGGCTGGAGCTGCTTC-3' & This work \\
\hline ppc-FW & $5^{\prime}$-CGCATCTTATCCGACCTACA-3' & This work \\
\hline ppc-RV & 5'-TGGCCTGTAGCAGAGTAGAG-3' & This work \\
\hline Plac-BamH-FW & 5'-ACATGGATCCGCGCCCAATACGCAAAC-3' & This work \\
\hline Plac-Pstl-RV & 5'-ACATCTGCAGAGCTGTTTCCTGTGTGAAAT-3' & This work \\
\hline Plac-Scal-RV & 5'-ACATAGTACTAGCTGTTTCCTGTGTGAAAT-3' & This work \\
\hline Fnr-FW & 5'-ACTACTGCAGATGATCCCGGAAAAGCGAATTA-3' & This work \\
\hline Fnr-RV & 5'-TCTATGCGCATCAGGCAACGTTACGCGTA-3' & This work \\
\hline modE-FW & 5'-ACTACTGCAGATGCAGGCCGAAATCCTTC-3' & This work \\
\hline modE-RV & 5'-TCTATGCGCATTAGCACAGCGTGGCGATA-3' & This work \\
\hline selc-FW & 5'-ACTACTGCAGATACGCTGCGTGTATTAGGC-3' & This work \\
\hline selC-RV & 5'-TCTATGCGCACTTAGACCAGGTATTCCTGAAG-3' & This work \\
\hline arcB-Xbal-FW & 5'-TCTAGATAAGGCCGCGGAGGATTACACTATGATGAAGCAAATTCGTCTGCT-3' & This work \\
\hline $\operatorname{arcB-Bgll-RV}$ & 5'-ATCTAGCCGGAAGGGCTCATTITTAGTGGCTITTGCC-3' & This work \\
\hline arcA-FW & 5'-ATGCAGACCCCGCACATTCT-3' & This work \\
\hline arcA-Xbal-RV & 5'-ACTATCTAGATTAATCTTCCAGATCACCGCA-3' & This work \\
\hline arcA-Xbal-RV & 5'-ACTATCTAGATTAATCTTCCAGATCACCGCA-3' & This work \\
\hline infB-Xbal-FW & 5'-TCTAGATAAGGCCGCGGAGGATTACACTATGATGACCAAGTCAGAATTGAT-3' & This work \\
\hline ihfB-Bgll-RV & 5'-ATCTAGCCGGAAGGGCTTAACCGTAAATATTGGCGC-3' & This work \\
\hline ihfA-FW & 5'-ATGGCGCTTACAAAAGCTGA-3' & This work \\
\hline ihfA-Xbal-RV & 5'-ACTATCTAGATTACTCGTCTTTGGGCGA-3' & This work \\
\hline
\end{tabular}

$12 \mathrm{~g} / \mathrm{L} \mathrm{K}_{2} \mathrm{HPO}_{4}, 3 \mathrm{~g} / \mathrm{L} \mathrm{KH}_{2} \mathrm{PO}_{4} ; \mathrm{pH}$ 7.6) or in M9 salts medium (Sigma-Aldrich) containing trace elements [35,36]. When required, kanamycin was added at a concentration of $30 \mu \mathrm{g} / \mathrm{ml}$. For studies requiring plasmid-directed gene expression, $1 \mathrm{mM}$ IPTG was added to the cultures 1 hour following inoculation of the fermentation medium, and induction was performed for approximately 4 hours.

Fermentations were carried out in $37 \mathrm{ml}$ serum vials (Wheaton) containing $15 \mathrm{ml}$ of TYP or M9 medium. Following the addition of medium, the vials were sealed using silicone stoppers and aluminum crimp seals (Supelco), and autoclaved. Prior to inoculation, the headspace was evacuated and flushed with Argon (ultra high purity, Airgas). Supplements included in TYP or M9 medium were: $0.5 \%$ or $1 \%(\mathrm{w} / \mathrm{v})$ glucose; $1 \mu \mathrm{M}$ sodium molybdate; $1 \mu \mathrm{M}$ sodium selenite; $50 \mu \mathrm{M}$ nickel sulfate. Approx- imately $1.3 \%(\mathrm{v} / \mathrm{v})$ of inoculum culture, prepared under aerobic conditions in TYP, was used to inoculate fermentation medium. Specific hydrogen yields were determined at approximately 5 hours, and 17 hours in TYP and M9 medium, respectively. Fermentations in $10 \mathrm{ml}$ of M9 medium containing $0.5 \%$ glucose $(\mathrm{w} / \mathrm{v})$ were run for 17 hours to determine the molar yields of hydrogen. The concentrations of all products generated from glucose were determined for strains cultured anaerobically for 34 hours in $15 \mathrm{ml}$ of M9 medium containing $1.0 \%$ glucose $(\mathrm{w} / \mathrm{v})$ to allow adequate time for the consumption of all of the glucose contained in the medium. All experiments were repeated six times with at least duplicate fermentations contained within each experiment. The $A_{600}$ for strains cultured anaerobically in M9 medium was measured by removing samples anoxically with a syringe in intervals for 34 hours. 


\section{Analytical methods}

The gas mixture from culture headspace was withdrawn using gastight syringes (Hamilton), and monitored for hydrogen formation. Hydrogen was detected using a Shimadzu GC-17A gas chromatograph (GC) equipped with a thermal conductivity detector (TCD), a 60/80 Molecular Sieve 5A column (Supelco), with argon as the carrier gas. The Clarity software package (DataApex) was used to analyze the chromatograms.

The formation of fermentation products were detected by high performance liquid chromatography (HPLC) using an Aminex HPX-87H ion exchange column (Bio-Rad; $7.8 \times 300 \mathrm{~mm}$ ) and a Varian ProStar 410 liquid chromatography instrument equipped with UV absorbance and refractive index detectors. Culture supernatant was filtered through $0.45 \mu \mathrm{M}$ cellulose acetate filters, and $40 \mu \mathrm{L}$ of sample was injected. The column was eluted isocratically with $5 \mathrm{mM} \mathrm{H}_{2} \mathrm{SO}_{4}$ at a flow rate of $0.5 \mathrm{ml} /$ minute. Data was analyzed using the Star Chromatography Workstation software. Ethanol was quantified using the Quantichrome assay kit

\section{References}

1. Buckley MaW, J (2006) Microbial Energy Conversion. American Academy of Microbiology Report.

2. Nath K, Das D (2004) Improvement of fermentative hydrogen production: various approaches. Appl Microbiol Biotechnol 65: 520-529.

3. Maeda T, Sanchez-Torres V, Wood TK (2007) Enhanced hydrogen production from glucose by metabolically engineered Escherichia coli. Applied Microbiology and Biotechnology 77: 879-890.

4. Yoshida A, Nishimura T, Kawaguchi H, Inui M, Yukawa H (2006) Enhanced hydrogen production from glucose using ldh- and frd-inactivated Escherichia coli strains. Appl Microbiol Biotechnol 73: 67-72.

5. Samir KumarKhanal, Wen-HsingChen, LingLi, Sung S (2004) Biological hydrogen production: effects of $\mathrm{pH}$ and intermediate products International. Journal of Hydrogen Energy 29: 1123-1131.

6. Gokarn RR, Eiteman MA, Altman E (2000) Metabolic analysis of Escherichia coli in the presence and absence of the carboxylating enzymes phosphoenolpyruvate carboxylase and pyruvate carboxylase. Applied and Environmental Microbiology 66: 1844-1850.

7. Ingraham JLaN FC (1987) Escherichia Coli and Salmonella Typhimurium: Cellular and Molecular Biology. ASM Press: 262-278.

8. Sawers G (1994) The hydrogenases and formate dehydrogenases of Escherichia coli. Antonie Van Leeuwenhoek 66: 57-88.

9. Sawers RG (2005) Formate and its role in hydrogen production in Escherichia coli. Biochemical Society Transactions 33: 42-46.

10. Suppmann B, Sawers G (1994) Isolation and characterization of hypophosphiteresistant mutants of Escherichia coli: identification of the FocA protein, encoded by the pfl operon, as a putative formate transporter. Mol Microbiol 11: 965-982.

11. Vignais PAea, FEMS Microbiology Reviews, 2001. 25: p. 455-501. (2001).

12. Self WT, Hasona A, Shanmugam KT (2004) Expression and regulation of a silent operon, hyf, coding for hydrogenase 4 isoenzyme in Escherichia coli. J Bacteriol 186: 580-587.

13. Salmon K, Hung SP, Mekjian K, Baldi P, Hatfield GW, et al. (2003) Global gene expression profiling in Escherichia coli K12. The effects of oxygen availability and FNR. J Biol Chem 278: 29837-29855.

14. Perrenoud A, Sauer U (2005) Impact of global transcriptional regulation by ArcA, ArcB, Cra, Crp, Cya, Fnr, and Mlc on glucose catabolism in Escherichia coli. J Bacteriol 187: 3171-3179.

15. Constantinidou C, Hobman JL, Griffiths L, Patel MD, Penn CW, et al. (2006) A reassessment of the FNR regulon and transcriptomic analysis of the effects of nitrate, nitrite, NarXL, and NarQP as Escherichia coli K12 adapts from aerobic to anaerobic growth. Journal of Biological Chemistry 281: 4802-4815.

16. Overton TWGL, Patel MD, Hobman JL, Penn CW, Cole JA, Constantinidou C (2006) Microarray analysis of gene regulation by oxygen, nitrate, nitrite, FNR, NarL and NarP during anaerobic growth of Escherichia coli: new insights into microbial physiology. Biochem Soc Trans 34: 104-107.

17. Self WT, Hasona A, Shanmugam KT (2001) N-terminal truncations in the FhlA protein result in formate- and MoeA-independent expression of the hyc (formate hydrogenlyase) operon of Escherichia coli. Microbiology-Sgm 147: 3093-3104.

18. Self WT, Grunden AM, Hasona A, Shanmugam KT (1999) Transcriptional regulation of molybdoenzyme synthesis in Escherichia coli in response to
(BioAssay Systems). Glucose was quantified by both HPLC (described above), and by the glucose oxidase (GO) assay kit (Sigma).

Dry cell mass was determined by filtration of the entire culture through a $0.8 \mu \mathrm{M}$ nitrocellulose membrane, drying the membrane at $60^{\circ} \mathrm{C}$ in a vacuum oven for about 14 hours, and determining the weight of the membrane.

\section{Acknowledgments}

We are grateful to Keith Kepler for technical assistance, and to Kenneth Zahn for insightful discussions.

\section{Author Contributions}

Conceived and designed the experiments: ZF LY RC. Performed the experiments: ZF RG. Analyzed the data: ZF LY RC. Contributed reagents/materials/analysis tools: ZF RC. Wrote the paper: RC. Revised manuscript drafts: ZF. Revised manuscript: LY.

molybdenum: ModE-molybdate, a repressor of the modABCD (molybdate transport) operon is a secondary transcriptional activator for the hyc and nar operons. Microbiology 145(Pt 1): 41-55.

19. Sawers RG (2005) Formate and its role in hydrogen production in Escherichia coli. Biochem Soc Trans 33: 42-46.

20. Hopper S, Babst M, Schlensog V, Fischer HM, Hennecke H, et al. (1994) Regulated expression in vitro of genes coding for formate hydrogenlyase components of Escherichia coli. J Biol Chem 269: 19597-19604.

21. Blokesch M, Paschos A, Theodoratou E, Bauer A, Hube M, et al. (2002) Metal insertion into NiFe-hydrogenases. Biochem Soc Trans 30: 674-680.

22. Yoshida A, Nishimura T, Kawaguchi H, Inui M, Yukawa H (2005) Enhanced hydrogen production from formic acid by formate hydrogen lyase-overexpressing Escherichia coli strains. Appl Environ Microbiol 71: 6762-6768.

23. Redwood MD, Mikheenko IP, Sargent F, Macaskie LE (2008) Dissecting the roles of Escherichia coli hydrogenases in biohydrogen production. FEMS Microbiol Lett 278: 48-55.

24. Maeda TS-TV, Wood TK (2007) Enhanced Hydrogen production from glucose by metabolically engineered Escherichia coli. Appl Microbiol Biotechnol 77: 879-890.

25. Adrio JL, Demain AL (2006) Genetic improvement of processes yielding microbial products. FEMS Microbiol Rev 30: 187-214.

26. Paulette MVignais, BernardBilloud, Meyer J (2006) Classification and phylogeny of hydrogenases. FEMS Microbiology Reviews 25: 455-501.

27. Perrenoud A, Sauer U (2005) Impact of global transcriptional regulation by ArcA, ArcB, Cra, Crp, Cya, Fnr, and Mlc on glucose catabolism in Escherichia coli. Journal of Bacteriology 187: 3171-3179.

28. Salmon K, Hung SP, et al. (2003) Global gene expression profiling in Escherichia coli K12. The effects of oxygen availability and FNR. J Biol Chem 278(32): 29837-55. 278: 29873-29855.

29. Maupin JA, Shanmugam KT (1990) Genetic regulation of formate hydrogenlyase of Escherichia coli: role of the fhlA gene product as a transcriptional activator for a new regulatory gene, fhlB. J Bacteriol 172: 4798-4806.

30. Schlensog V, Lutz S, Bock A (1994) Purification and DNA-binding properties of FHLA, the transcriptional activator of the formate hydrogenlyase system from Escherichia coli. J Biol Chem 269: 19590-19596.

31. http://biocyc.org/ECOLI/NEW-IMAGE?type $=$ ENZYME\&object $=$ NARLMONOMER.

32. http://biocyc.org/ECOLI/NEW-IMAGE?type $=$ ENZYME\&object $=$ PD00197.

33. Petri R, Schmidt-Dannert C (2004) Dealing with complexity: evolutionary engineering and genome shuffling. Curr Opin Biotechnol 15: 298-304.

34. Datsenko KA, Wanner BL (2000) One-step inactivation of chromosomal genes in Escherichia coli K-12 using PGR products. Proc Natl Acad Sci U S A 97: 6640-6645.

35. Miller JH (1972) Experiments in Molecular Genetics Cold Spring Harbor, Cold Spring Harbor, NY.

36. Jacobi A, Rossmann R, Bock A (1992) The hyp operon gene products are required for the maturation of catalytically active hydrogenase isoenzymes in Escherichia coli. Arch Microbiol 158: 444-451. 